\title{
REVISORES QUE CONTRIBUYERON EN ESTE NÚMERO
}

\author{
Jésica Aquino \\ María Florencia Bono \\ Juan Manuel Busso \\ José Alejandro Di Conza \\ Gabriela Echeverría \\ Diego Eiras \\ Daniel González Acuña \\ Mariano Mastropaolo \\ Daniel Durán Osorio \\ Estefanía Perez \\ Andrea Racca \\ Ermelinda Rivera \\ Marcelo Signorini \\ Pablo Siroski \\ Alejandro Soraci \\ José Manuel Venzal
}

